Article

\title{
Two Flavors of Hydrogen Atoms: A Possible Explanation of Dark Matter
}

\section{Eugene Oks}

Physics Department, Auburn University, 380 Duncan Drive, Auburn, AL 36849, USA; goks@physics.auburn.edu

Received: 11 March 2020; Accepted: 30 June 2020; Published: 6 July 2020

\begin{abstract}
In one of our previous papers, it was shown that for the ground state of hydrogenic atoms/ions, it is possible to match the interior (inside the nucleus) solution of the Dirac equation with the singular exterior solution of the Dirac equation, so that the singular solution should not be rejected for the ground state of hydrogenic atoms/ions. In that paper, there was presented also the first experimental proof of the existence of this Alternative Kind of Hydrogen Atoms (AKHA) - by showing that the presence of the AKHA solves a long-standing mystery of the huge discrepancy between the experimental and previous theoretical results concerning the high-energy tail of the linear momentum distribution in the ground state of hydrogen atoms. In another paper, we showed that for hydrogen atoms, the singular solution of the Dirac equation outside the proton is legitimate not just for the ground state $1^{2} S_{1 / 2}$, but also for the states $2^{2} S_{1 / 2}, 3^{2} S_{1 / 2}$ and so on: it is legitimate for all the discrete states $n^{2} S_{1 / 2}$. Moreover, the singular exterior solution is legitimate also for the $1=0$ states of the continuous spectrum. In that paper, we demonstrated that the AKHA can be the basis for explaining the recent puzzling astrophysical observational results concerning the redshifted radio line $21 \mathrm{~cm}$ from the early Universe. Thus, there seems to be the astrophysical evidence of the existence of the AKHA-in addition to the already available observational proof of their existence from atomic experiments. In the present paper, we point out that the AKHA provide an alternative view on dark matter-without resorting to new subatomic particles or dramatically changing the existing physical laws. This is because due to the selection rules, the AKHA do not have state that can be coupled by the electric dipole radiation. We also reformulate the above theoretical results in terms that hydrogen atoms can have two flavors: one flavor corresponding to the regular solution outside the proton, another- to the singular solution outside the proton, both solutions corresponding to the same energy. Since this means the additional degeneracy, then according to the fundamental theorem of quantum mechanics, there should be an additional conserved quantity, which we call isohydrogen spin (isohyspin). Further atomic experiments for accurately measuring the high-energy tail of the linear momentum distribution in the ground state of hydrogen atoms, as well as further observational studies of the redshifted $21 \mathrm{~cm}$ radio line from the early Universe, could provide a further proof that dark matter or a part of it is the AKHA.
\end{abstract}

Keywords: alternative kind of hydrogen atoms; two flavors of hydrogen atoms; new symmetry of hydrogen atoms; explanation of the dark matter; explanation of the puzzle of $21 \mathrm{~cm}$ radio line; early Universe

\section{Introduction}

Hydrogenic atoms/ions are the simplest quantum systems and were always considered as a test-bed for checking quantum theories versus observations. There existed a paradigm that, even with the allowance for the finite nuclear size, singular solutions of the Dirac equation for the Coulomb problem should be rejected for nuclear charges $Z<1 / \alpha\left(\alpha=\mathrm{e}^{2} /(\hbar \mathrm{c})\right.$ being the fine structure constant $)$ 
not only for the excited states, but even for the ground state of hydrogen atoms/hydrogenlike ions (hereafter, GSHA) ${ }^{1}$. In our previous [1], this paradigm was broken as follows.

First, there was derived a general condition for matching a regular solution inside the nucleus with a singular exterior solution of the Dirac equation for arbitrary spherically symmetric interaction potential $\mathrm{V}(\mathrm{r})$, which takes two different forms in the interior region $\mathrm{r}<\mathrm{R}$ and in the exterior region $\mathrm{r}>\mathrm{R}$, where $\mathrm{R}$ is the nuclear size. This was an important general result in its own right. For the models of the proton charge density, such as the spherical shell charge density or the uniform charge density (used in the literature before [1]), the corresponding interior solutions cannot be matched with the singular exterior solution of the Dirac equation for the Coulomb field even for the GSHA. However, these models were unrealistic: from experiments on the elastic scattering of electrons on protons (see, e.g., [2-4]) it is well known that the charge density inside proton neither has a peak at the periphery (as for the spherical shell charge density) nor is constant (as for the uniform charge density), but rather it has a maximum at $\mathrm{r}=0$.

Second, it was shown in [1] that for spherically symmetric interior potentials, corresponding to realistic charge densities having the maximum at $r=0$, it is possible to match the interior solution with the singular exterior solution of the Dirac equation for the Coulomb field for the GSHA. This means that the singular solution of the Dirac equation for the Coulomb field should not be rejected for the GSHA.

The motivation for [1] was to explain the long-standing mystery of the high-energy tail of the linear momentum distribution in the GSHA (below, for brevity we use simply the word "momentum" meaning "linear momentum"). Fock [5] derived the following distribution of the momentum $d w=f(p) d p$ for the bound electron in the GSHA, where the high-energy tail of the momentum distribution (HTMD) function $\mathrm{f}(\mathrm{p})$ scales $\propto 1 / \mathrm{p}^{6}$. However, for hydrogen atoms the experiments seem to favor an HTMD $\sim 1 / \mathrm{p}^{\mathrm{k}}$, where $\mathrm{k}$ is at least 1.5 times smaller than in Equation (2)—according to the detailed discussion in [1].

In [1], it was shown that the engagement of the singular solution for the GSHA yields for the HTMD an effective power law $\mathrm{f}^{\mathrm{As}}(\mathrm{p}) \propto 1 / \mathrm{p}^{\mathrm{k}}$ with the value of $\mathrm{k}$ noticeably smaller than 6 , thus resolving the above long-standing mystery. This fact constitutes the experimental proof that there are indeed hydrogen atoms in the ground state described by the singular solution of the Dirac equation outside the proton.

In [6], it was pointed out that for hydrogen atoms, the singular solution of the Dirac equation outside the proton is legitimate not just for the ground state $1^{2} S_{1 / 2}$, but also for the states $2^{2} S_{1 / 2}, 3^{2} S_{1 / 2}$ and so on: it is legitimate for all the discrete states $n^{2} S_{1 / 2}$. Moreover, the singular exterior solution is legitimate also for the $1=0$ states of the continuous spectrum.

We also point out that this Alternative Kind of Hydrogen Atoms (AKHA) can be the basis for explaining the puzzling astrophysical observational results by Bowman et al. [7], as shown in detail in [6] - without introducing an unspecified dark matter. The existence of the AKHA provides also an alternative, more natural view on dark matter-without resorting to new subatomic particles or dramatically changing the existing physical laws.

In the present paper, as the logical continuation of the existence of AKHA, we offer further considerations resulting from this fact. They deal with a new additional symmetry and the corresponding new additional conserved quantity.

\section{Brief Summary of the Results of our Previous Papers}

The Dirac equation yields the following eigenvalues of the energy of hydrogen atoms in the stationary states are (see, e.g., [8]):

$$
\mathrm{E}_{\mathrm{Nk}}=\mathrm{mc}^{2}\left\{1+\alpha^{2} /\left[\mathrm{N}+\left(\mathrm{k}^{2}-\alpha^{2}\right)^{1 / 2}\right]^{2}\right\}^{-1 / 2}
$$

1 The commonly accepted "regular" solution has a weak singularity at the origin. Here and below, by "singular" we mean the strongly-singular solution of the Dirac equation for the Coulomb field. 
where $\mathrm{N}$ is the radial quantum number and $\mathrm{k}$ is the eigenvalue of the operator $\mathrm{K}=\beta(2 \mathrm{Ls}+1)$, where $\beta$ is the Dirac matrix of the rank four and Ls denotes the scalar product of the operators of the orbital angular momentum and spin. For the GSHA, the quantum numbers $\mathrm{N}$ and $\mathrm{k}$ have the values $\mathrm{N}=0$, $\mathrm{k}=-1$, so that $\mathrm{E}_{0,-1}=\mathrm{mc}^{2}\left(1-\alpha^{2}\right)^{1 / 2}$.

The radial part $R_{\mathrm{Nk}}(\mathrm{r})$ of the coordinate wave functions for hydrogen atoms has the following form at small $r$ (see, e.g., [8]):

$$
\mathrm{R}_{\mathrm{Nk}}(\mathrm{r}) \propto 1 / \mathrm{r}^{1+\mathrm{s}}, \mathrm{s}= \pm\left(\mathrm{k}^{2}-\alpha^{2}\right)^{1 / 2}
$$

For the GSHA, Equation (2) simplifies to:

$$
\mathrm{R}_{0,-1}(\mathrm{r}) \propto 1 / \mathrm{r}^{\mathrm{q}}, \mathrm{q}=1 \pm\left(1-\alpha^{2}\right)^{1 / 2}
$$

In [1], it was shown that with the allowance for the finite proton size, both the regular exterior solution corresponding to $q=1-\left(1-\alpha^{2}\right)^{1 / 2}$ and the singular exterior solution corresponding to $q=1+\left(1-\alpha^{2}\right)^{1 / 2}$ are legitimate for the GSHA.

The derivation in [1], used only the fact that in the ground state the eigenvalue of the operator $\mathrm{K}$ is $\mathrm{k}=-1$. Therefore, actually, the derivation from [1] is valid not just for the ground state, but for any state of hydrogenic atoms/ions corresponding to the quantum number $\mathrm{k}=-1$, as pointed out in [6]. Such states are the S-states $(1=0)$, specifically the ${ }^{2} S_{1 / 2}$ states. So, not only the regular exterior solution corresponding to $\mathrm{q}=1-\left(1-\alpha^{2}\right)^{1 / 2}$, but also the singular exterior solution corresponding to $\mathrm{q}=1+\left(1-\alpha^{2}\right)^{1 / 2}$ is legitimate not just for the ground state $1^{2} S_{1 / 2}$, but also for the states $2^{2} S_{1 / 2}, 3^{2} S_{1 / 2}$ and so on. In other words, both the regular solution and the singular solution are legitimate for the states $n^{2} S_{1 / 2}$, where $\mathrm{n}=\mathrm{N}+|\mathrm{k}|=\mathrm{N}+1$ is the principal quantum number $(\mathrm{n}=1,2,3, \ldots)$.

Moreover, both the regular exterior solution corresponding to $q=1-\left(1-\alpha^{2}\right)^{1 / 2}$ and the singular exterior solution corresponding to $q=1+\left(1-\alpha^{2}\right)^{1 / 2}$ are legitimate also for the $1=0$ states of the continuous spectrum. This is because, for hydrogen atoms, the radial part of the coordinate wave functions of the continuous spectrum has the same behavior at small $r$, given by Equation (2), as for the discrete spectrum.

\section{Possible Explanation of Dark Matter}

From astrophysical observations, it is clear that in the Universe there is over five times more of the unknown, dark matter than the known, ordinary matter. Existing hypotheses about dark matter either introduce new, undiscovered subatomic particles or propose to dramatically change the known physical laws—see, e.g., reviews [9-12] and references therein.

We suggest that dark matter or a part of it could be represented by the AKHA. This is due to the fact that the AKHA does not have discrete or continuous states that could be coupled via the electric dipole radiation. Indeed, all states of the AKHA correspond to $1=0$, so that such coupling is prohibited by the selection rules. This idea provides a more natural explanation of dark matter: it does not require introducing new, undiscovered subatomic particles or dramatically changing the known physical laws.

Moreover, the existence of the AKHA in the Universe could explain a puzzling observational result published in Nature by Bowman et al. [7] where the authors observed the hydrogen $21 \mathrm{~cm}$ line (redshifted from the rest frequency of $1240 \mathrm{MHz}$ to the frequency of $78 \mathrm{MHz}$ ) from the early Universe. The puzzling result by Bowman et al. [7] was that the amplitude of the observed absorption profile was more than a factor of two greater than the largest predictions. This could indicate that the primordial hydrogen gas was much cooler than expected from the standard cosmology.

Several possible astrophysical explanations of the result by Bowman et al. [7] were published, such as, e.g., papers [13,14], where the authors resorted to an unspecified dark matter as the cooling agent. In [6], we provided what seems to be a more natural explanation. The AKHA would participate in the absorption signal of the redshifted line $21 \mathrm{~cm}$ : the ground state of the AKHA has the same 
superfine structure as the ground state of usual hydrogen atoms ${ }^{2}$. However, because the excited discrete and continuous states of the AKHA cannot be coupled by the electric dipole radiation, then in the course of the Universe expansion the AKHA decouple from the cosmic microwave background earlier than usual hydrogen atoms. As a result, the AKHA fluid is cooler than the fluid of usual hydrogen atoms. In [6], it was shown that this provides not only the qualitative explanation, but also the quantitative explanation of the puzzling observational result from Bowman et al. [7].

\section{Two Flavors of Hydrogen Atoms}

Below is a logical continuation of the above fundamental results. On the one hand, for hydrogen atoms both the regular ground state and the singular ground state correspond to the same energy given by Equation (3) with the same quantum numbers $\mathrm{N}=0, \mathrm{k}=-1$. In other words, the ground state of hydrogen atoms, in addition to the trivial double-degeneracy with respect to the $\mathrm{z}$ - projection $\mathrm{m}_{\mathrm{j}}$ of the total angular momentum J, has an additional double-degeneracy ${ }^{3}$.

On the other hand, there is a fundamental theorem of quantum mechanics concerning the cause of any additional degeneracy. Namely, it is caused by the existence of an additional conserved quantity (or quantities), whose operator commutes with the Hamiltonian, but does not commute with other conserved quantities or if it does, but the additional conserved quantity is a multi-component one, then its components do not commute with each other-see, e.g., [15]. The corresponding degenerate states differ by the eigenvalues (i.e., by additional quantum numbers) of the additional conserved quantity. For example, in the nonrelativistic Coulomb problem, the higher than geometrical degeneracy (the geometrical one being due to the spherical symmetry of the problem) is caused by the existence of the Runge-Lenz vector $\mathbf{A}$, whose operator commutes with the Hamiltonian, but does not commute with some components of the angular momentum operator (and the components of the Runge-Lenz vector operator do not commute with each other)—see, e.g., [15]. For a given energy, the corresponding nonrelativistic degenerate states differ not only by the eigenvalue of the operator $\mathrm{L}_{\mathrm{z}}$, but also by the eigenvalue of the operator $\mathrm{A}_{\mathrm{z}}$.

However, according to [1], the GSHA is double-degenerate despite these two states having the same values of all the known conserved quantities $\mathrm{E}, \mathrm{K}, \mathrm{J}^{2}$ and $\mathrm{J}_{\mathrm{z}}$. This means that there should be an additional, new conserved quantity, by eigenvalues of which these two ground states differ. In other words, the situation is that hydrogen atoms have two flavors, differing by the eigenvalue of an additional, new conserved quantity: hydrogen atoms have flavor symmetry.

Speaking of flavors, let us recall by analogy that quarks have flavors: for example, there are up and down quarks. For representing this particular flavor symmetry, there was assigned an operator of the isotopic spin (isospin) I-the operator having two eigenvalues for its z-projection: $I_{z}=1 / 2$ assigned to the up quark and $I_{z}=-1 / 2$ assigned to the down quark.

Therefore, it seems reasonable to introduce here a new operator: the operator of isohydrogen spin, abbreviated as isohyspin and denoted as $\mathrm{I}^{(\mathrm{h})}$. Similarly to the isospin, the z-projection of the isohyspin operator has two eigenvalues: $\mathrm{I}^{(\mathrm{h})}{ }_{\mathrm{z}}=1 / 2$ assigned to the regular flavor of hydrogen atoms and $\mathrm{I}^{(\mathrm{h})} \mathrm{z}=-1 / 2$ assigned to the singular flavor of hydrogen atoms.

\footnotetext{
For this reason, rigorously speaking, the AKHA could be called the nearly-dark matter.

To avoid any confusion, we remind the following to the readers. For the case of the corresponding Schrödinger equation, the ground state is non-degenerate as the consequence of the so-called "oscillation theorem". This theorem proves that the ground state wave function has no nodes, from where it follows that the ground state is non-degenerate-see. e.g., the textbook [15]. However, in the case of the Dirac equation, there need not be a nodeless eigenfunction for the ground state-because the oscillation theorem for the Dirac equation differs from the oscillation theorem for the Schrödinger equation-see, e.g., paper [16]. Physically this difference is due to the fact that for the Schrödinger equation there is a lower bound for the discrete energy spectrum, while there is no lower bound in the case of the Dirac equation - because it allows infinite number of solutions of the energy $\mathrm{E}<-\mathrm{mc}^{2}$.
} 
The isospin couples to the strong force (strong interaction). This is logical because it is related to intra-nuclear physics, where the strong interaction plays the dominant role. As a result, the strong force can transform the up quark into the down quark and vice versa.

In distinction, the isohyspin does not relate to intra-nuclear physics: so, it would be logical to state that the isohyspin does not couple to the strong force/interaction-since the isohyspin relates to a hydrogen atom as the whole. For the same reason, it would be logical to state that the isohyspin does not couple to the weak force/interaction. Additionally, there seems no ground to expect that the isohyspin would couple to the gravitational force/interaction. As for the electromagnetic force/interaction, the (ordinary) spin couples to the magnetic field, but the isospin does not couple to the electromagnetic force/interaction. Therefore, there seems to be no reason for the isohyspin to couple to the electromagnetic force/interaction either.

It should be emphasized that the ideas presented in this section are not necessary for considering the AKHA as the candidate for the dark matter or for a part of the dark matter.

\section{Conclusions}

We significantly expanded the fundamental results of our previous papers [1,6]. Since the AKHA do not have excited states that can be coupled with the electric dipole radiation. Therefore they could represent dark matter (or a nearly dark matter) or a part of it. In this way, dark matter or a part of it could be explained without resorting to new subatomic particles or without dramatically changing the physical laws ${ }^{4}$.

The AKHA can be the basis for explaining the puzzling astrophysical observational results by Bowman et al. [7], as shown in [6]—-without introducing an unspecified dark matter. Thus, there seems to be the astrophysical evidence of the existence of the AKHA-in addition to the already available observational proof of their existence from atomic experiments (the latter being shown in [1]).

We reformulated the above theoretical results in terms that hydrogen atoms can have two flavors: one flavor corresponding to the regular solution outside the proton, another-to the singular solution outside the proton. Since this means the additional degeneracy, then according to the fundamental theorem of quantum mechanics, there should be an additional conserved quantity, which we called isohydrogen spin (isohyspin) $I^{(h)}$. The eigenvalues of the operator $I^{(h)}$ are $+1 / 2$ for the regular flavor and $-1 / 2$ for the singular flavor of hydrogen atoms. The isohyspin does not seem to couple to any of the four known fundamental forces/interactions ${ }^{5}$.

We hope that the results of the present paper would motivate further atomic experiments for accurately measuring the high-energy tail of the linear momentum distribution in the ground state of hydrogen atoms, as well as further observational studies of the redshifted $21 \mathrm{~cm}$ radio line from the early Universe. The latter could provide further proof that the dark matter or a part of it is the AKHA.

Funding: This research received no external funding.

Conflicts of Interest: The author declares no conflict of interest.

4 Electric quadrupole radiation is allowed for the AKHA, but it is extremely weak. We note that electric quadrupole transitions would occur without changing the flavor (and thus the value of the isohyspin) of hydrogen atoms. Observations of electric quadrupole transitions in atoms and molecules require a very sophisticated technique (using, in particular, multiple lasers simultaneously) and were published only recently-see, e.g., papers [17,18]. Such experiments with hydrogen atoms were not reported in the literature, to the best of our knowledge.

5 Hypothetical radiative transitions between the states of the AKHA and the states of the usual hydrogen atoms would require the "flip" of the isohyspin from the state of the eigenvalue $-1 / 2$ to the state of the eigenvalue $+1 / 2$. As long as this effect is not observed, this should indicate a very low transition probability for the isohyspin flip. 


\section{References}

1. Oks, E. High-energy tail of the linear momentum distribution in the ground state of hydrogen atoms or hydrogen-like ions. J. Phys. B At. Mol. Opt. Phys. 2001, 34, 2235-2243. [CrossRef]

2. Simon, G.G.; Schmitt, C.; Borkowski, F.; Walther, V.H. Absolute electron-proton cross sections at low momentum transfer measured with a high pressure gas target system. Nucl. Phys. A 1980, 333, 381-391. [CrossRef]

3. Perkins, D.H. Introduction to High Energy Physics; Addison-Wesley: Menlo Park, CA, USA, 1987.

4. Feynman, R.P. Photon-Hadron Interactions; Lecture 24; Addison-Wesley: Redwood City, CA, USA, 1989.

5. Fock, V. Zur Theorie des Wasserstoffatoms. Z. Phys. 1935, 98, 145-154. [CrossRef]

6. Oks, E. Alternative Kind of Hydrogen Atoms as a Possible Explanation of the Latest Puzzling Observation of the $21 \mathrm{~cm}$ Radio Line from the Early Universe. Res. Astron. Astrophys. 2020, 20, 109.

7. Bowman, J.D.; Rogers, A.E.E.; Monsalve, R.A.; Mozdzen, T.J.; Mahesh, N. An absorption profile centred at 78 megahertz in the sky-averaged spectrum. Nature 2018, 555, 67-70. [CrossRef] [PubMed]

8. Rose, M.E. Relativistic Electron Theory; Wiley: New York, NY, USA, 1961.

9. Bertone, G.; Tait, T.M.P. A new era in the search for dark matter. Nature 2018, 562, 51-56. [CrossRef] [PubMed]

10. Bertone, G.; Hooper, D. History of dark matter. Rev. Mod. Phys. 2018, 90, 045002. [CrossRef]

11. Garrett, K.; Düda, G. Dark Matter: A Primer. Adv. Astron. 2011, 2011, 968283. [CrossRef]

12. Majumdar, D. Dark Matter: An Introduction; CRC Press: Boca Raton, FL, USA, 2014.

13. Barkana, R. Possible interaction between baryons and dark-matter particles revealed by the first stars. Nature 2018, 555, 71-74. [CrossRef] [PubMed]

14. Tashiro, H.; Kadota, K.; Silk, J. Effects of dark matter-baryon scattering on redshifted $21 \mathrm{~cm}$ signals. Phys. Rev. D 2014, 90, 083522. [CrossRef]

15. Landau, L.D.; Lifshitz, E.M. Quantum Mechanics; Pergamon: Oxford, UK, 1965.

16. Rose, M.E.; Newton, R.R. Properties of Dirac wave functions in a central field. Phys. Rev. 1951, 82, 470. [CrossRef]

17. Ponciano-Ojeda, F.; Hernandez-Gomez, S.; Lopez-Hernandez, O.; Mojica-Casique, C.; Colın-Rodrıguez, R.; Ramirez-Martinez, F.; Flores-Mijangos, J.; Sahagun, D.; Jauregui, R.; Jimenez-Mier, J. Observation of the 5 $p 3 / 2 \rightarrow 6$ p3/2 electric-dipole-forbidden transition in atomic rubidium using optical-optical double-resonance spectroscopy. Phys. Rev. A 2015, 92, 042511. [CrossRef]

18. Germann, M.; Tong, X.; Willitsch, S. Observation of electric-dipole-forbidden infrared transitions in cold molecular ions. Nat. Phys. 2014, 10, 820-824. [CrossRef] 